\title{
Composición Isotópica del Agua Subterránea y su Relación con la Salinidad en el Valle de San Luis Río Colorado en Sonora, México
}

\author{
Lorenzo Valenzuela $^{(1)}$, Jorge Ramírez-Hernández $^{(2)}$ y Ramón B. Palomares ${ }^{(1)}$ \\ (1) Universidad Estatal de Sonora, Km. 6.5 Carretera a Sonoita, San Luis Río Colorado 83450, \\ Sonora-México (e-mail: valenzuela_lorenzo@yahoo.com) \\ (2) Universidad Autónoma del Estado de Baja California, Instituto de Ingeniería, Ave. De la Normal s/n \\ Col. Insurgentes-Este Mexicali, 21280 B.C.-México (e-mail: jorger@uabc.edu.mx)
}

Recibido Sep. 11, 2012; Aceptado Nov. 05, 2012; Versión final recibida Ene. 04, 2013

\begin{abstract}
Resumen
En este trabajo, se analiza la composición isotópica del acuífero del Valle de San Luis Río Colorado en Sonora en conjunto con la concentración de cloruros del agua para determinar el origen del agua del subsuelo y establecer las causas del aumento de salinidad que se ha observado en el agua de riego. La mineralización del acuífero ha provocado un deterioro en la calidad del agua de esta agua. Se encontró que la composición isotópica deja ver un fraccionamiento como consecuencia de la evaporación rápida y la mezcla de aguas antiguas, infiltradas antes de la construcción del sistema de presas, con aguas recientes del Río Colorado, sometidas a un proceso de evaporación en los vasos de éstas. El análisis isotópico permitió establecer que el agua de los canales de riego más mineralizada aún no está presente en el acuífero.
\end{abstract}

Palabras claves: isótopos estables, acuífero, agua subterránea, calidad de agua, evaporación

\section{Isotopic composition of groundwater and its relationship with salinity in the Valley of San Luis Rio Colorado Sonora Mexico.}

\begin{abstract}
In this work, the isotopic composition and chloride concentration of San Luis Rio Colorado, Sonora, in Mexico were used to determine the origin of groundwater and to establish the salinity increase that has been observed in the irrigation water. The aquifer mineralization has provoked deterioration of this water. The isotopic composition showed a fractioning due to the sudden evaporation and the mixing of water percolated before the dam system, constructed upstream, with water infiltrated to the aquifer after evaporation in the reservoirs, these water types are known as old and recent, respectively. Unlike what it could be expected, the isotopic composition determined that mineralized water from irrigation channels is not yet present in the aquifer.
\end{abstract}

Keywords: stable isotopes, aquifer, groundwater, water quality, evaporation 


\section{INTRODUCCIÓN}

La actividad agrícola del Valle de San Luis Río Colorado en Sonora, perteneciente al Distrito de Riego 014, se desarrolla en una superficie aproximada de 26,400 ha con derecho de riego, donde son irrigadas 14,000 ha con aguas subterráneas provenientes de la explotación de aproximadamente 167 pozos profundos y 12,400 ha con aguas superficiales conducidas por gravedad, cuya fuente de abastecimiento común es el Río Colorado a través de la presa "Morelos" y el canal de riego Sánchez Mejorada. El Distrito de Riego No. 14 está dividido para su administración en Módulos de Riego. Al Valle de San Luis Río Colorado le corresponden los Módulos de Riego No. 1, 2 y 3. El Módulo No. 1 está ubicado en la margen izquierda del Rio Colorado, entre las coordenadas $32^{\circ} 28^{\prime} 36^{\prime \prime}$ latitud Norte y $114^{\circ} 45^{\prime} 45^{\prime \prime}$ longitud Oeste y forma parte del municipio que lleva el mismo nombre el cual está localizado al noroeste de la República Mexicana en el Estado de Sonora con una extensión territorial de $8,412.75 \mathrm{~km}^{2}$, (Fig. 1).

El agua tanto del sistema de flujo superficial como del subterráneo en el Delta del Río Colorado tiene su origen en el agua que aporta el Río Colorado, la aportación por lluvia en el Delta es muy reducida, la precipitación media anual en la estación Yuma Valley de la red AZMET es de $80 \mathrm{~mm}$, (AZMET, 2012). No obstante, la salinidad del agua entregada superficialmente a México por el Tratado Internacional de 1944 ha sufrido variaciones en su salinidad alcanzando niveles inaceptables para los usos agrícolas en los años 70s, ya que es mezclada con agua de retorno agrícola que ha sufrido procesos de evaporación y lavado de suelos.

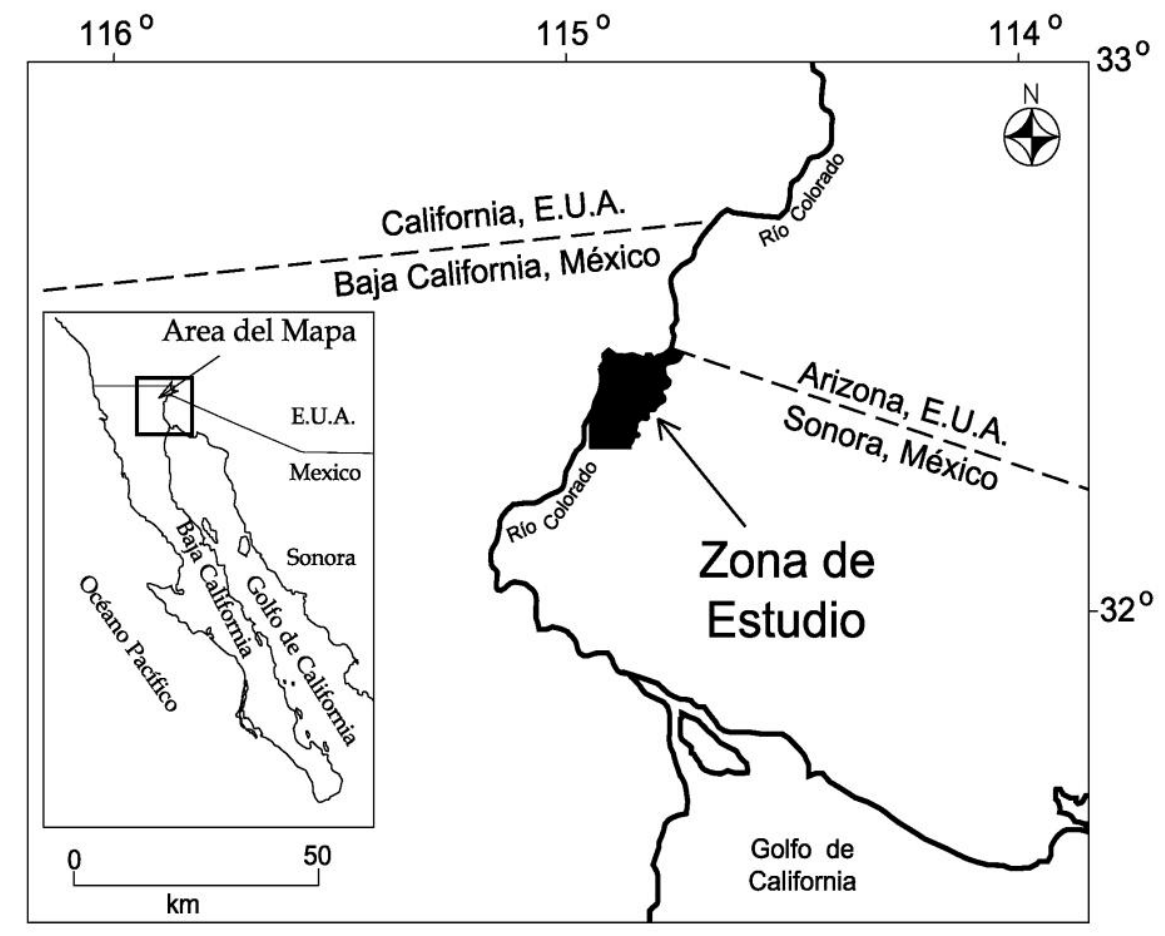

Fig. 1 Localización del Módulo de Riego No. 1, del Valle de San Luis Río Colorado Sonora, del Distrito de Riego No. 014.

La minuta 242 firmada en 1973 impidió, a partir de entonces, la entrega de agua con niveles de salinidad mayores a 121 partes por millón, en promedio anual, a el agua de la presa Morelos y que es entregada a los usuarios agrícolas del Valle Imperial en California, EUA, (IBWC, 1973). No obstante, la salinidad de las aguas de riego, con valores de conductividad eléctrica entre 1,140 y $3,820 \mu \mathrm{Sm}^{-1}$ con promedio de 2,123 $\mu \mathrm{S} \mathrm{cm}^{-1}$, utilizadas para los cultivos son uno de los principales problemas que ha afectado la producción agrícola en el valle de San Luis R.C. desde hace más de 5 décadas, (CONAGUA, 2008; Getches, 2003; IBWC, 1973).

El agua que históricamente fluía a lo largo del cauce del Río Colorado aportó sedimentos y formó los valles de Imperial y Mexicali conformando el delta. El agua infiltrada a lo largo de su recorrido alimentó el acuífero. A partir de la construcción del sistema de presas aguas arriba del Río Colorado, en 1934 (inicio del llenado del vaso de la presa Hoover), el caudal fue drásticamente regulado, (Roman-Calleros y Ramírez-Hernández, 2003). La firma del Tratado Internacional de 1944 los volúmenes que eran descargados a México fueron acotados a $1,850 \times 10^{6} \mathrm{~m}^{3}$ anuales, (CILA, 1944). La residencia del agua en los vasos de almacenamiento 
favorece la evaporación y por lo tanto el fraccionamiento isotópico, lo que permite diferenciar el agua subterránea infiltrada antes de haber sido almacenada en estos vasos, (Tompson et al., 2008).

Valenzuela-Vasquez et al. (2009) encontraron que en la mayoría de los pozos del Módulo de Riego No. 1 las aguas son clasificadas como cloruradas sódicas de acuerdo con el diagrama de Piper. Además, en los pozos ubicados al norte del Módulo de Riego No. 1, observaron que las aguas subterráneas se dirigen del Noreste al Suroeste atravesando toda el área del módulo siguiendo el cauce del Río Colorado. Dada la complejidad del comportamiento de las aguas de los pozos del Módulo de Riego No. 1 y las dificultades para definir los factores determinantes del incremento de sales mediante modelación hidrogeoquímica recomiendan determinar la composición isotópica del agua, la cual se definiría el origen. La composición isotópica del agua en conjunto con las modelaciones hidrogeoquímicas permitiría clarificar los diferentes aportes de agua a los pozos del Módulo de Riego No.1.

El objetivo del presente trabajo es identificar los orígenes de las fuentes del agua subterránea del acuífero por medio del estudio de la composición isotópica de los isótopos estables $\delta_{2} \mathrm{H}$ (Deuterio, D) y $\delta_{18} \mathrm{O}(\mathrm{Oxígeno}$ 18) que permitan explicar el aumento de la salinidad del agua y el suelo, para que las medidas preventivas y de mitigación aplicadas sean más efectivas y se logre un mejor manejo del agua.

\section{ANTECEDENTES}

Las técnicas isotópicas se han aplicado en hidrología e hidrogeología a nivel mundial durante más de 50 años, constituyéndose en una herramienta muy valiosa para la investigación y la solución de problemas relacionados con el medio ambiente y la ingeniería,(Fontes, 1986; Gonfiantini, 1986). La utilización de los isótopos estables del agua, $\delta_{2} \mathrm{H}$ y $\delta_{18} \mathrm{O}$, son muy útiles para identificar las diferentes fuentes de recarga de agua subterráneas y distinguir aguas con una composición química similar, ya que son trazadores ideales porque son parte de la molécula de agua y no reaccionan como otros elementos disueltos en el agua (Dickinson et al., 2006).Por una parte, la concentración absoluta y relativa de los iones mayoritarios y traza en el agua reflejan los procesos de interacción agua-roca. Por otra, la composición isotópica de las aguas subterráneas pueden utilizarse para localizar las zonas de recarga, y para determinar el origen de las aguas subterráneas (meteórico, agua marina, fósil, magmática y metamórfica) (Fontes, 1986).

La molécula de agua está formada por dos elementos: hidrógeno y oxígeno. El hidrógeno se presenta en la naturaleza bajo la forma de dos isótopos estables, el protio $\left({ }_{1} \mathrm{H}\right)$ y el deuterio $\left({ }_{2} \mathrm{H}\right.$ o $\left.\mathrm{D}\right)$, y uno radiactivo, el tritio $\left({ }_{3} \mathrm{H}\right.$ O T). El oxígeno posee tres isótopos estables: ${ }_{16} \mathrm{O},{ }_{17} \mathrm{O}$ y ${ }_{18} \mathrm{O}$. La relación ${ }_{18} \mathrm{O} /{ }_{16} \mathrm{O}$ es de aproximadamente 1:500 y la ${ }_{2} \mathrm{H} /{ }_{1} \mathrm{H}$ de 1:6600 en el agua del océano (Plata, 1996)

En 1961, Harmon Craig encuentra una relación lineal entre la composición isotópica de $\delta_{18} \mathrm{O}$ y la de $\delta_{2} \mathrm{H}$ para las aguas superficiales a escala global y la denomina "Craig's global meteoricwater line" (Línea de Agua Meteórica Global de Craig), representada por la siguiente ecuación:

$\delta_{2} \mathrm{H}=8 \delta_{18} \mathrm{O}+10 \%$ smow

donde $\delta_{2} \mathrm{H}$ es la relación isotópica de Deuterio y $\delta_{18} \mathrm{O}$ es la relación isotópica de oxígeno-18.

En la figura 2 se muestra la Línea Meteórica Mundial comparada con los cambios de la composición isotópica del agua producida por diferentes procesos. Siendo que el estado líquido del agua en el mar es uno de los pasos del ciclo hidrológico, y que este contiene el $98 \%$ del agua líquida presente en la superficie de la tierra, su composición promedio se ha tomado como el estándar o patrón internacional (SMOW, Standard Mean OceanWater) para el análisis de información isotópica. Toda concentración de isótopos estables en la molécula de agua se presenta entonces como la desviación delta $(\delta)$ con referencia al patrón antes mencionado, de la siguiente manera:

$\delta=\left(\mathrm{R}-\mathrm{R}_{\text {SMOW }}\right) / \mathrm{R}_{\text {SMOW }}$

Donde $\mathrm{R}$ es la llamada relación isotópica y representa el cociente entre los isótopos estables, ya sea del hidrógeno $\left({ }_{2} \mathrm{H} /{ }_{1} \mathrm{H}\right)$ o del oxígeno $\left({ }_{18} \mathrm{O} /{ }_{16} \mathrm{O}\right)$.

Posteriormente es introducido el patrón VSMOW (Viena Standard Mean OceanWater) que es el utilizado actualmente. El subsiguiente monitoreo isotópico de la precipitación mundial realizado por la Organismo Internacional de Energía Atómica por sus siglas en ingles GNIP-OIEA, a través de la red global para isótopos en la precipitación, ha refinado esta relación obteniendo la siguiente expresión(Vélez y Rhenals, 2008): 
$\delta^{2} H=8.13 \delta_{18} \mathrm{O}+10.8 \mathrm{VSMOW}$

Los resultados obtenidos con isotopos estables demuestran que elenfoque multi-isotópico es una herramienta útil para identificar el origen y las fuentes de aguas subterráneas salinas (Jørgensen y Banoeng-Yakubo, 2001). Siendo los isotopos ${ }_{18} \mathrm{O} / 2 \mathrm{H}$ buenos trazadores de movimiento del agua y de algunos procesos de fraccionamientos naturales que les afectan.

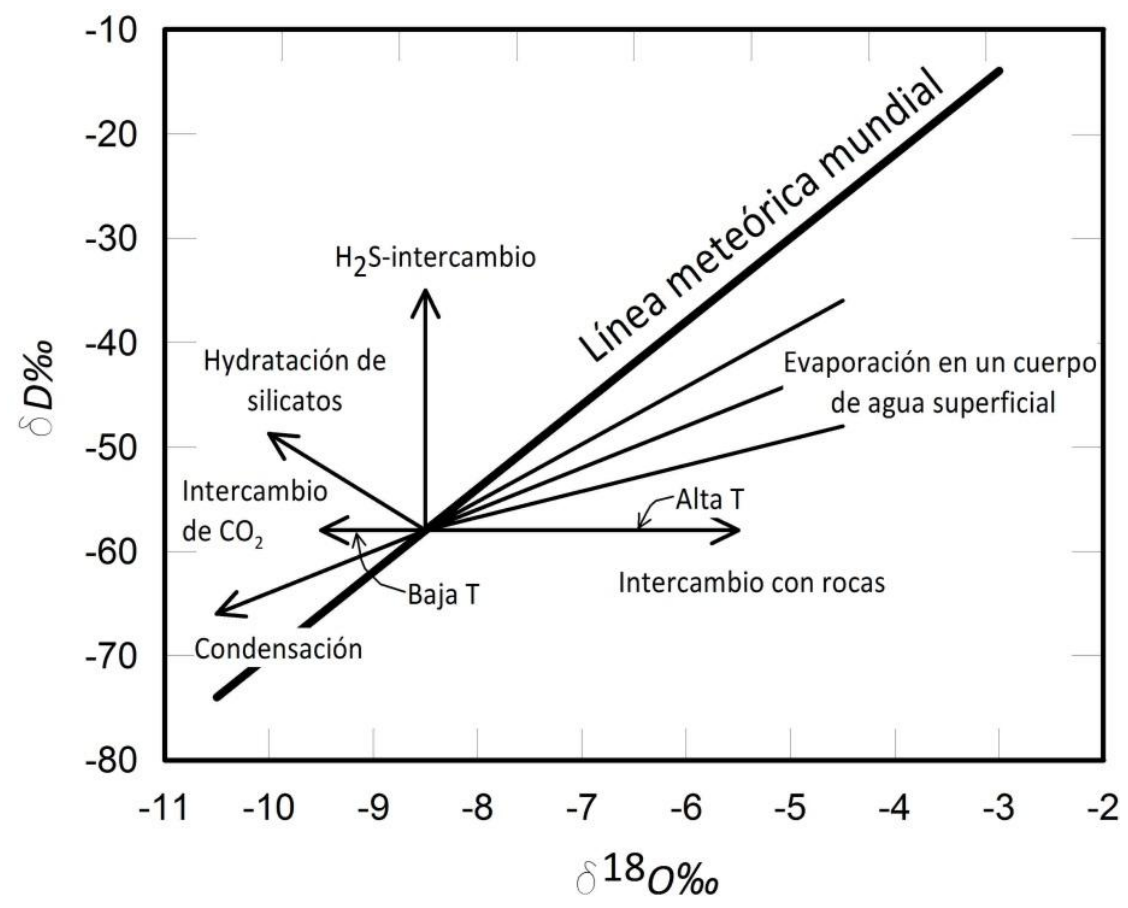

Fig. 2. Línea Meteórica mundial comparada con los cambios de la composición isotópica del agua producida por diferentes procesos. Modificada de (Domenico y Schwartz, 1998).

La variación relativa de la composición isotópica de $\delta_{18} \mathrm{O}$ con respecto a $\delta_{2} \mathrm{H}$ cuando el agua ha estado sometida a un proceso de evaporación da como resultado líneas con pendientes entre 4 y 6 con valores de la ordenada menores a 10, (Domenico y Schwartz, 1998; Mebus, 2000; Plata, 1996). La composición isotópica del agua subterránea en regiones áridas puede ser en ocasiones, bastante diferente de la composición de la precipitación local, indicando que las causas más comunes es el enriquecimiento isotópico del agua por evaporación.

De otra parte, para interpretar los resultados de los análisis isotópicos del agua es necesario tener parámetros de referencia, uno de ellos es la línea meteórica mundial, la otra es la línea meteórica del cauce que se desarrolla en el espacio orográfico del área de estudio y la línea meteórica local; también es de importancia tener de referencia líneas meteóricas de áreas circundantes. Tompson et al., (2008) utilizaron como referencia la línea meteórica mundial (global meteoricwater line) y la línea meteórica del Río Colorado para interpretar sus resultados, en esta última se observan dos nubes de puntos, la nube desplazada hacia los valores mayores de composición isotópica expresan, según el autor, un origen reciente del agua del Río Colorado (entre 10 a 15 años de edad), compuesto por agua primordialmente evaporada debido a un mayor tiempo de exposición atmosférica de la misma, la nube de puntos localizada en valores menos agotados del tritio (isótopo radiactivo) representan aguas antiguas del río de aproximadamente 45 años de edad, que pertenecieron a etapas anteriores a la construcción de las grandes presas aguas arriba. (Fig. 3).

Schroeder et al., (1991) encontraron que el agua afectada por la evaporación, como sucede en el riego, se muestran en una mayor agotamiento de deuterio que de ${ }_{18} \mathrm{O}$. La composición isotópica del agua, una vez en el acuífero, no cambia tan fácilmente, excepto, posiblemente, por la mezcla con otra fuente de distinto valor isotópico, estos es un indicativo de la presencia de agua superficial aportada a través del riego, (Fontes, 1986). Rangel-Medina et al., (2004)destacan que, a partir de los valores isotópicos $\delta_{18} \mathrm{O}$ y $\delta_{2} \mathrm{H}$ del agua subterránea del acuífero que recarga el Río Sonora y la Línea Meteórica Mundial, este acuífero presenta un grado de afectación por una evaporación rápida y desértica, típica de una infiltración reciente y sujeta a variaciones estacionales. A partir de los resultados obtenidos con isotopos estables el enfoque isotópico es una herramienta útil para identificar el origen y las fuentes de aguas subterráneas salinas resultando los 
isotopos $\delta_{18} \mathrm{O}$ y $\delta_{2} \mathrm{H}$ buenos trazadores de movimiento del agua y de algunos procesos de fraccionamientos naturales que les afectan (Gonfiantini, 1986; Jørgensen y Banoeng-Yakubo, 2001). La composición isotópica del agua subterránea en regiones áridas puede ser en ocasiones, bastante diferente de la composición de la precipitación local, indicando que la causa más común es el enriquecimiento isotópico del agua por evaporación.

El modelo hidrogeológico del acuífero del delta del Río Colorado corresponde a un potente acuífero libre detrítico compuesto de sedimentos acarreados por el Río Colorado en su porción central (600 a 4000 m de profundidad), intercalado con sedimentos de piedemonte de las montañas que limitan al delta en el flanco Oeste con las Sierras Cucapah y El Mayor en el flanco Este con las montañas Laguna, Gila y Las Tinajas (CONAGUA, 2008). Su flujo regional es en dirección SW desde las inmediaciones de las Sierras Chocolate entre las que discurre el Río Colorado hasta la cabecera del Golfo de California. Estos flujos regionales son modificados localmente por la extracción de agua de pozos para uso agrícola y por recarga vertical desde canales de riego sin revestimiento y agua de retorno de riego agrícola. Particularmente en esta porción del acuífero los canales sin recubrimiento más importantes son el Canal Bacastec y el Canal Revolución, (CONAGUA, 2008).

Palomares (2011),en modelación hidrogeoquímica de los procesos de mineralización del agua de los pozos del Módulo No. 1, utilizando el código PRHEEQC, a lo largo de tres líneas de flujo, encontró que: 1) los pozos ubicados a lo largo del cauce del Río Colorado podrían contener agua que procede de agua reciente del mismo Río y que su evolución hidrogeoquímica pasa de aguas cloruradas sódicas a sulfatadas sódicas; 2) el agua de los pozos de la porción central del Módulo No. 1 demostró que es posible que se lleve a cabo un intercambio catiónico incorporándose sodio y precipitándose calcio y magnesio por el contacto con depósitos de halita en el subsuelo; 3) el agua de los pozos ubicados en el borde Este del Módulo No. 1, cercanos a la mesa arenosa, muestran una evolución hacia aguas cloruradas sódicas.

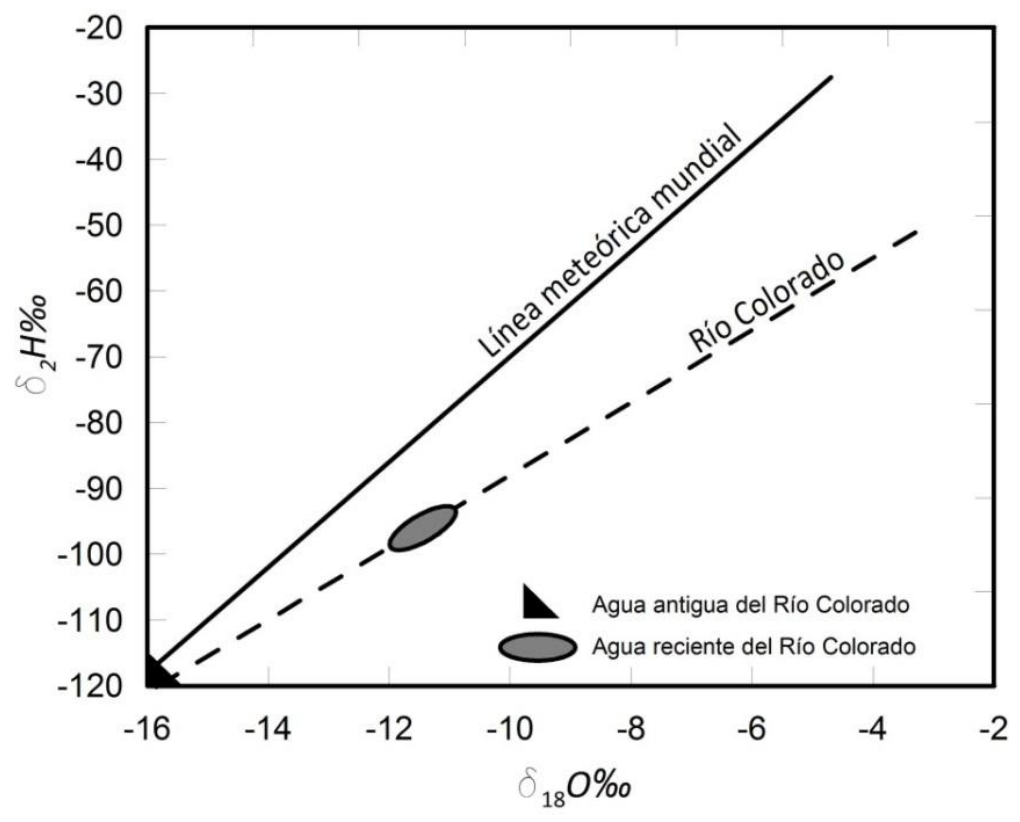

Fig. 3. Composición isotópica del agua del Río Colorado propuesta por Tompson et al. (2008), comparada con la Línea Meteórica Mundial propuesta por Craig (1961).

\section{MATERIALES Y MÉTODOS}

Se tomaron muestras de agua de 34 pozos del Módulo de Riego No. 1 en los meses de marzo y abril del año 2009 (Fig. 4). Para cada pozo se utilizaron tres botellas de polietileno reforzado de $250 \mathrm{ml}$, con tapones especiales para evitar la mínima presencia de aire en el depósito. La primera botella para cationes con ácido nítrico al $10 \%$, la segunda para analizar aniones y la tercera para analizar isótopos. Además se tomaron muestras en dos puntos del canal Sánchez Mejorada y una más del agua subterránea proveniente de la Mesa de Andrade o Mesa Arenosa. En total se colectaron 102 muestras, representativas del área de estudio (Fig. 1), las muestras se transportaron en hieleras y durante su almacenamiento se guardaron en un refrigerador a una temperatura mínima de $4^{\circ} \mathrm{C}$, como lo recomienda el Manual de Geoquímica Ambiental de la (UNAM, 2005). 
Al momento de tomar las muestras de agua referidas en el párrafo anterior, se hicieron las siguientes determinaciones "in situ". Se registraron las coordenadas geográficas de ubicación de cada pozo utilizando un GPS marca Garmin, modeloLegend. La conductividad eléctrica (CE) y los sólidos disueltos totales (STD) del agua colectada se midió con un conductímetro portátil marca Hanna, modelo HI 9835. La temperatura y el $\mathrm{pH}$ del agua se evaluó con un potenciómetro portátil marca Jenko. Además se midió la profundidad del nivel estático en cada pozo con una sonda eléctrica marca Solinst.

Los iones mayoritarios de las muestras de agua se analizaron en el laboratorio de Ciencias de la Tierra del Instituto de Ingeniería de la Universidad Autónoma de Baja California utilizando lo metodología de la APHAAWWA-WPFC (1992), se obtuvo la concentración de los siguientes iones: Calcio $\left(\mathrm{Ca}^{+2}\right.$, Sodio $\left(\mathrm{Na}^{+1}\right)$, Potasio $\left(\mathrm{K}^{+1}\right)$, Magnesio $\left(\mathrm{Mg}^{+2}\right)$, Carbonatos $\left(\mathrm{CO}_{3}^{-2}\right)$, Bicarbonatos $\left(\mathrm{HCO}_{3}^{-1}\right)$, Sulfatos $\left(\mathrm{SO}_{4}^{-2}\right)$, Cloro $\left(\mathrm{Cl}^{-1}\right)$ y Sílice $\left(\mathrm{SiO}_{4}\right)$. Los análisis de la composición isotópica de $\delta_{18} \mathrm{O}$ y Deuterio $\left(\delta_{2} \mathrm{H}\right)$ se obtuvieron en el laboratorio EnvironmentallsotopeLaboratory del Department of Geosciences de la Universidad de Arizona en Tucson Arizona, EUA.

El análisis de la composición isotópica se llevó a cabo mediante la comparación gráfica con la Línea Meteórica Mundial corregida en 1973, de la Viena Estándar Mean OceanWater (VSMOW) (Craig, 1961; Vélez y Rhenals, 2008)y la Línea de Evaporación del Río Colorado utilizada por Tompson et al. (2008) lo que permitió determinar el trazo y origen del agua en el acuífero del Valle de San Luis Río Colorado, Sonora.

\section{RESULTADOS Y DISCUSION}

Los resultados de los análisis isotópicos se presentan en la Tabla 1. La figura 4 ilustra las isopiezas que muestran un gradiente desde la porción NE en los pozos R-58 y R-36 de $20 \mathrm{msnm}$ hasta la isopieza de 14 msnm en la porción SW en el pozo 23-Valle. Este gradiente general en dirección SW obedece a la recarga regional del Río Colorado desde aguas arriba de la zona de estudio, como se ha indicado por diversos autores, (CONAGUA, 2008).

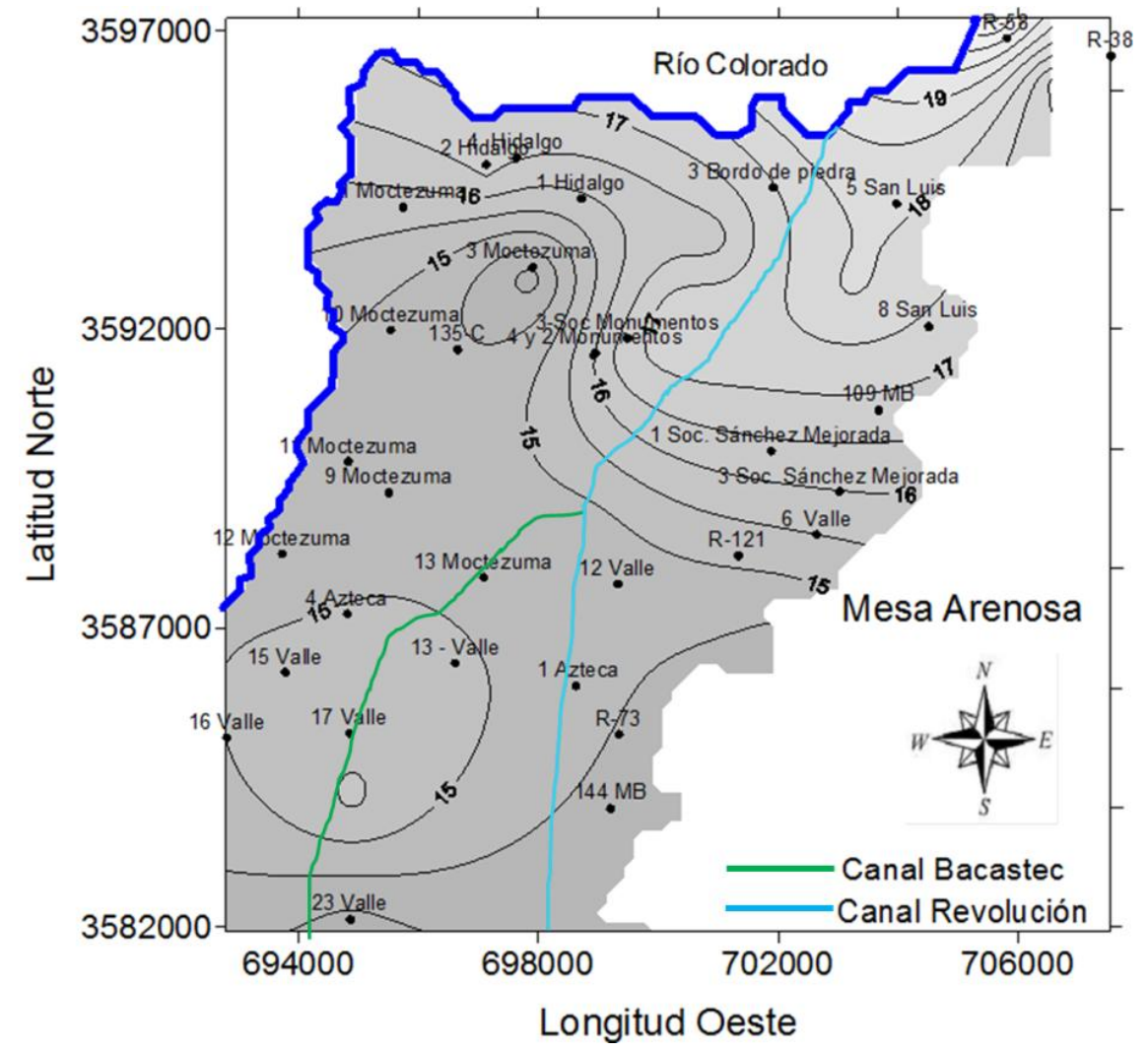

Fig. 4. Ubicación e identificación de los 34 pozos muestreados e isolíneas del nivel piezométrico (isopiezas) de los pozos del Módulo de Riego No. 1 con separación de $0.5 \mathrm{~m}$. Las líneas en azul corresponden al trazo del Río Colorado, en la frontera Norte y Noroeste, Canal Revolución con orientación NE-SW que se divide dos en la porción central del área de estudio en Canal Bacastec. Las coordenadas en proyección UTM, WGS85. 
Tabla 1. Resultados de los análisis de isótopos y cloruros del agua subterránearealizados en el mes abril del 2009.

\begin{tabular}{|l|c|c|c|l|c|c|c|}
\hline Nombre del Pozo & $\delta \mathrm{D} \%$ & $\delta_{18} \mathrm{O} \%$ & $\begin{array}{c}\mathrm{Cl} \\
\left(\mathrm{mg} \mathrm{L}^{-1}\right)\end{array}$ & Nombre del Pozo & $\delta \mathrm{D} \%{ }^{\circ}$ & $\delta_{18} \mathrm{O} \%$ & $\begin{array}{c}\mathrm{Cl} \\
\left(\mathrm{mg} \mathrm{L}^{-1}\right)\end{array}$ \\
\hline 2 Hidalgo & -94 & -11.8 & 380 & 13 Moctezuma & -110 & -14.3 & 199 \\
\hline 1 Moctezuma & -103 & -13.3 & 364 & 12 Valle & -108 & -13.9 & 247 \\
\hline 109 MB & -106 & -13.8 & 341 & 6 Valle & -109 & -14.1 & 235 \\
\hline 3 Bordo de Piedra & -95 & -12.0 & 353 & 15 Valle & -107 & -13.9 & 260 \\
\hline 5 San Luis & -95 & -11.8 & 355 & 16 Valle & -107 & -13.8 & 455 \\
\hline 8 San Luis & -108 & -14.2 & 281 & 17 Valle & -110 & -14.6 & 160 \\
\hline 1 Hidalgo & -96 & -11.9 & 210 & 11 Moctezuma & -104 & -13.4 & 688 \\
\hline R-38 & -108 & -14.3 & 229 & 4 Hidalgo & -99 & -12.4 & 270 \\
\hline 10 Moctezuma & -112 & -14.7 & 220 & 3 Moctezuma & -108 & -14.0 & 394 \\
\hline 2 Monumentos & -111 & -14.5 & 133 & 23 Valle & -109 & -14.2 & 257 \\
\hline 135-C & -107 & -13.9 & 646 & 144 MB & -107 & -14.0 & 422 \\
\hline 3-Soc Monumentos & -105 & -13.4 & 434 & R-58 & -96 & -12.0 & 358 \\
\hline 4 Monumentos & -109 & -14.0 & 401 & R-121 & -107 & -14.0 & 397 \\
\hline 1 Soc. Sánchez Mejorada & -103 & -14.2 & 233 & R-73 & -109 & -14.2 & 305 \\
\hline 3 Soc. Sánchez Mejorada & -105 & -13.5 & 553 & $13-$ Valle & -109 & -14.2 & 312 \\
\hline 12 Moctezuma & -104 & -13.3 & 501 & 1 Azteca & -104 & -13.6 & 314 \\
\hline 9 Moctezuma & -110 & -14.3 & 401 & 4 Azteca & -110 & -14.4 & 156 \\
\hline
\end{tabular}

La composición isotópica de agua en el gráfico $\delta_{18} \mathrm{O}$ vs $\delta_{2} \mathrm{H}$ muestran una pendiente de 6.61 y la coordenada en el origen de -22.86. Esta línea de evaporación inicia en el agua de origen (antigua) que corresponde al agua infiltrada del Río Colorado antes de la construcción del sistema de presas, aguas arriba. La composición isotópica del agua en el acuífero indica una mezcla de agua recientemente evaporada (retorno de riego) con agua antigua y agua actual del Río Colorado presentada de acuerdo a los valores presentados por Tompson et al. (2008), (Fig. 5).

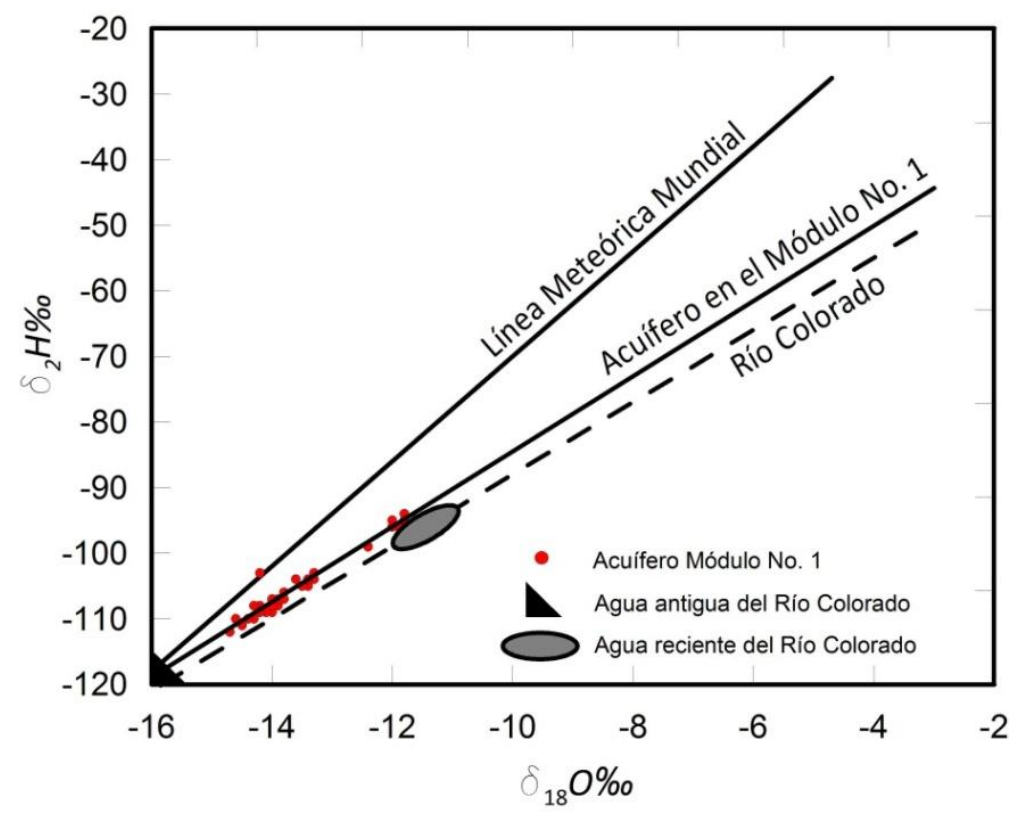

Fig. 5. Composición isotópica del agua del acuífero en el Módulo No. 1 obtenida en este trabajo comparada con la Línea Meteórica Mundial corregida en 1973 (Vélez y Rhenals, 2008) y la línea del agua del Río Colorado obtenida por Tompson et al. (2008).

Los pozos que se ubican en el área cercana al cauce del Río Colorado y al norte del Módulo No. 1 presentan valores mayores en la composición del $\delta_{18} \mathrm{O}$ y del $\delta_{2} \mathrm{H}$, como se aprecia en las figuras 6 y 7 , respectivamente. Existe posible mezcla del agua reciente del Río Colorado en el agua subterránea, siendo más notorio en los pozos localizados al norte del Módulo No.1. Los pozos ubicados en la parte central y al Este del área de estudio, presentan menores valores en la composición isotópica, esta área puede estar influenciada por agua de origen del Río Colorado (antigua), mezclada con agua evaporada producto de la infiltración del riego. 


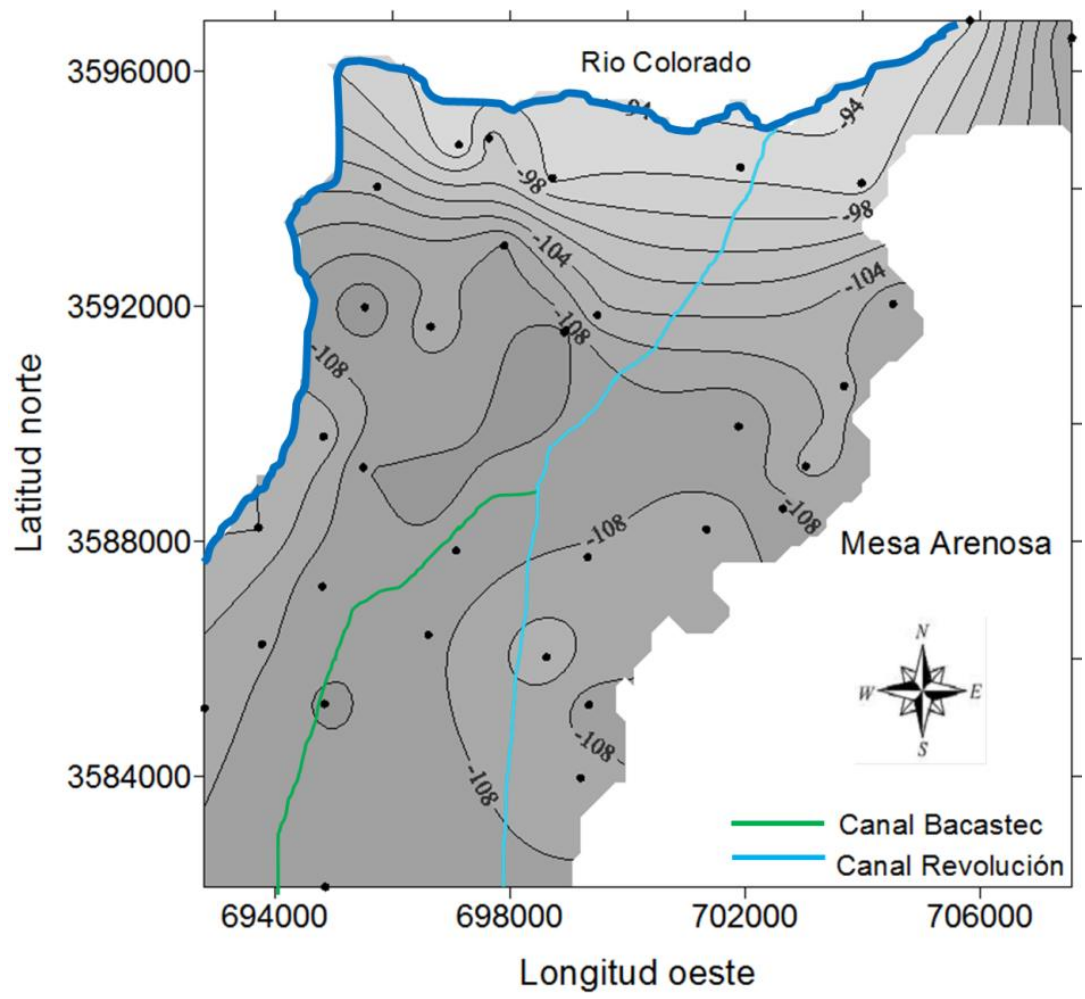

Fig. 6. Distribución espacial de la composición de $\delta_{18} \mathrm{O}$, en el agua subterránea de los pozos del Módulo de Riego No. 1.

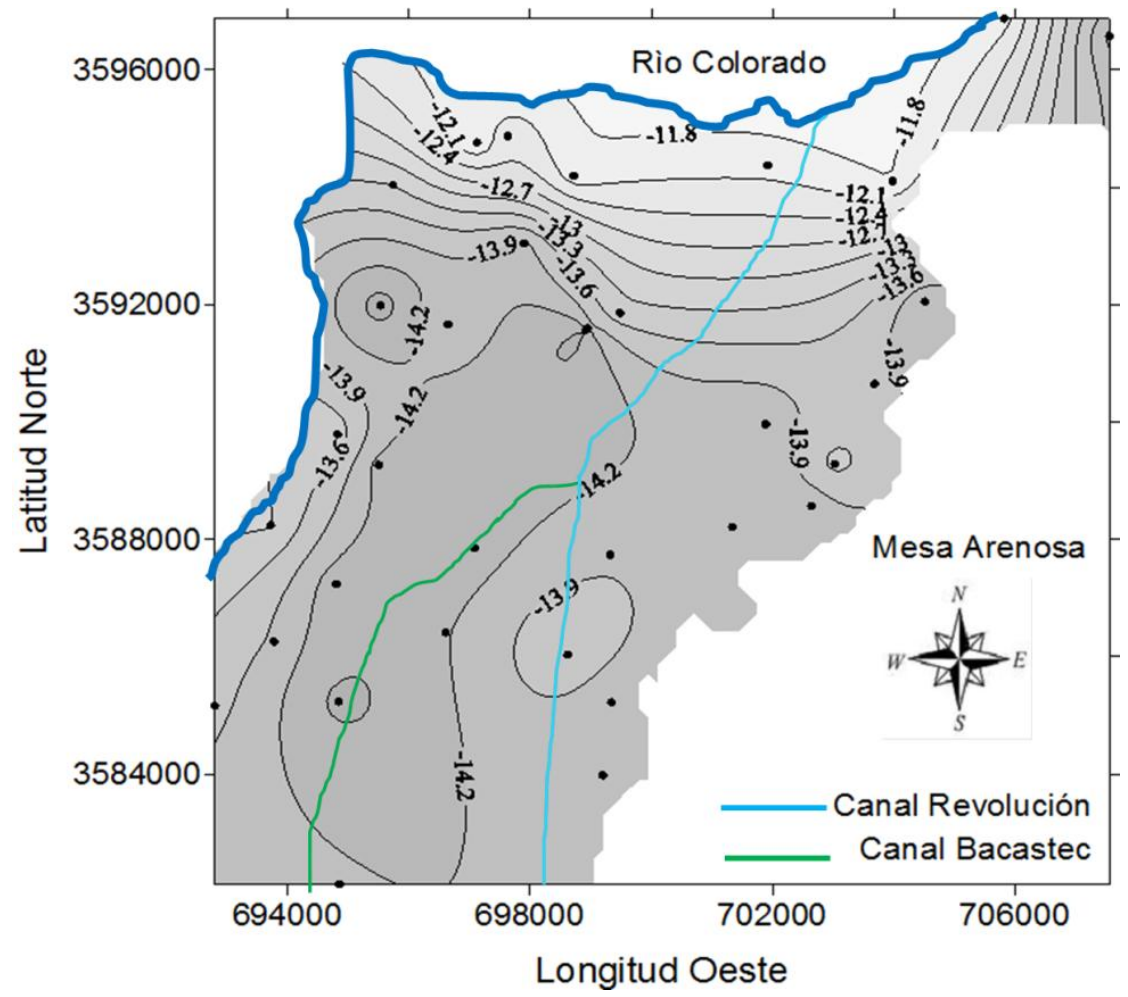

Fig. 7. Distribución espacial de la composición de $\delta_{2} \mathrm{H}$, en el agua subterránea de los pozos del Módulo de Riego No. 1.

La concentración de cloruro se produce por un proceso de disolución de sales presentes en el suelo y en el subsuelo, en la figura 8 se aprecia este proceso de disolución, indicando con una elipse a los pozos que poseen una composición isotópica de $\delta_{18} \mathrm{O}$ menor a $-12.5 \%$. Este grupo de 6 pozos de composición isotópica menor que el resto de los pozos, independientemente de la concentración de cloruro, son: 2 Hidalgo, 5 San Luis, R-58, 3 Bordo de Piedra, 4 Hidalgo y 1 Hidalgo. En la figura 4 se observa que todos 
ellos se localizan en el área Norte del Módulo de Riego No. 1, lo cual podría indicar la influencia de una mezcla de flujo de agua más reciente y se puede corroborar en la figura 5, donde el mismo grupo de pozos se localizan exactamente sobre el área del agua reciente del RC desplazados sobre la línea meteórica del Módulo de Riego No. 1.

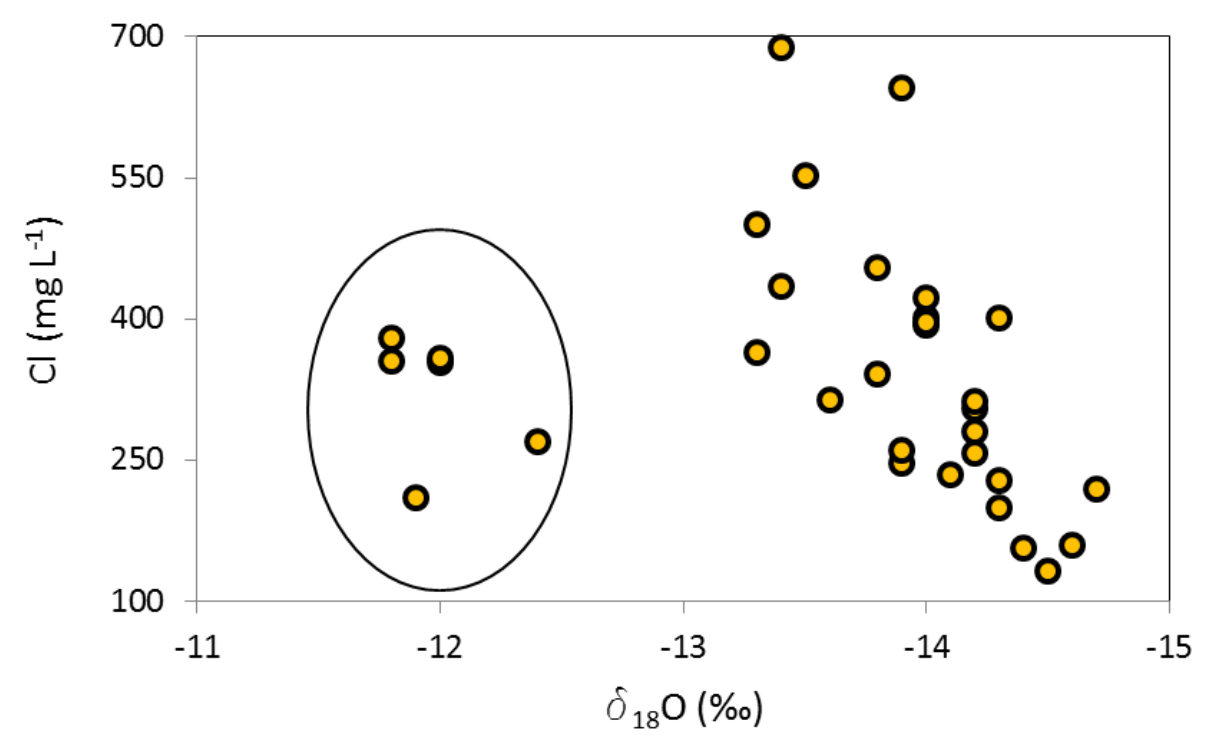

Fig. 8. Relación entre la concentración de cloruros y la composición isotópica del $\delta_{18} \mathrm{O}$ en partes por mil.

\section{CONCLUSIONES}

El agua subterránea en el Módulo de Riego No. 1 mostró una composición isotópica $\left(\delta_{18} \mathrm{O}\right.$ y $\left.\delta_{2} \mathrm{H}\right)$ característica de aguas originadas antes de la construcción del sistema de presas (aguas antiguas del Río Colorado) y de aguas infiltradas después de la construcción del sistema sometidas a procesos de evaporación en los vasos (aguas recientes del Río Colorado). Particularmente, en la parte norte del Módulo de Riego No. 1, se encontraron dos procesos de mezcla: uno, agua proveniente de infiltración reciente del Río Colorado mezclada con agua evaporada debido a un mayor tiempo de exposición atmosférica de la misma; dos, agua reciente con agua antigua del Río Colorado. Por la concentración de cloruros no se atribuye una componente de agua infiltrada desde el Canal Bacastec.

A diferencia de lo que se esperaba, el análisis isotópico permitió establecer que el agua de los canales de riego más mineralizada aún no está presente en el acuífero lo cual permite establecer políticas del uso del agua que impidan su contaminación. Con lo anterior, se demuestra que el análisis de la composición isotópica del agua es una herramienta útil en el manejo de los recursos hídricos en una zona semidesértica.

\section{REFERENCIAS}

AZMET, The Arizona Meteorological Network (en línea) http://ag.arizona.edu/azmet/02.htm. Acceso: 25 de Septiembre (2012).

CILA, Tratado entre el Gobierno de los Estados Unidos Mexicanos y el Gobierno de los Estados Unidos de America de la distribucion de las Aguas Internacionales de los Rios Colorado, Tijuana y Bravo, desde Fort Quitman , Texas, hasta el Golfo de Mexico, 1944. (en linea), http://www.sre.gob.mx/cilanorte/images/stories/pdf/1944.pdf. Acceso: 4 de Enero (1944).

CONAGUA, Determinación de la Disponibilidad de Agua en el Acuífero 2601 Valle de San Luis Río Colorado, Estado de Sonora. 2008.20 (en http://www.conagua.gob.mx/OCNO07/Noticias/2601\%20Valle\%20de\%20San\%20Luis\%20R\%C3\%ADo\%20 Colorado.pdf. Acceso: 4 de Enero (2013).

Craig, H., Isotopic Variations in Meteoric Waters, Science, 133 (3465), 1702-1703 (1961).

Dickinson, J.E., Land, M., Faunt, C.C., Leake, S.A., Reichard, E.G., Fleming, J.B. y Pool , D.R., Hydrologic framework refinement, ground-water flow and storage, water-chemistry analyses, and water-budget 
components of the Yuma area, Southwestern Arizona and Southeastern California. Reston, Virginia. 2006, Scientific Investigation Report 2006-5135. (en linea), http://pubs.usgs.gov/sir/2006/5135/. Acceso: 6 de Noviembre (2012).

Domenico, P.A. y Schwartz, F.W., Physical and Chemical Hidrogeology, $2^{\text {a }}$ edición, John Wiley \& Sons, Inc., (1998).

Fontes, J.C., Environmental isotopes in groundwater hydrology, in Handbook of environmental isotope geochemistry, Vol I by Fritz P.y Fontes J.C., pp 75-140 Elsevier, Amsterdam, Netherlands (1986).

Getches, D.H., Water Management in the United States and the Fate of the Colorado River Delta in Mexico, US-Mexico Law Journal, 11, 107-113 (2003).

Gonfiantini, R., Environmental isotopes in lake studies, in Handbook of environmental isotope geochemistry, Vol 2 by Fritz P.y Fontes J.C., pp 113-168 Elsevier, Amsterdam, Netherlands (1986).

IBWC, Minute No. 242-Permanent and definitive solution to the international problem of the salinity of the Colorado River, 1973. (en linea), http://www.ibwc.gov/Files/Minutes/Min242.pdf, Acceso: 5 de Diciembre (2013).

Jørgensen, N. y Banoeng-Yakubo, B., Environmental isotopes $\left({ }^{18} \mathrm{O},{ }^{2} \mathrm{H}\right.$, and $\left.{ }^{87} \mathrm{Sr}{ }^{86} \mathrm{Sr}\right)$ as a tool in groundwater investigations in the Keta Basin, Ghana, Hydrogeology Journal, 9 (2), 190-201 (2001).

Mebus, G., Groundwater. Saturated and unsaturated zone, in: Environmental isotopes in the hydrological cycle Principles and applications, by Mook W.G. UNESCO Paris, International Hydrological Programme. UNESCO and IAEA, IHP-V Technical Documents in Hydrology No. 39, Vol. IV, pp. 196 (2000).

Palomares, R.R., Identificación de los componentes hidrogeoquimicos que contaminan el acuífero del módulo de riego 01 del valle de San Luis Rio Colorado, Sonora,Tesis de Maestría en Ingeniería, Instituto de Ingeniería, Universidad Autonoma de Baja California, Mexicali, Baja California, México (2011).

Plata, B.A., Generalidades sobre isótopos estables del agua. Curso sobre Hidrología, 1a Edición. Universidad Autónoma de Madrid, España, (1996).

Rangel-Medina, M., Monreal, S.R., Morales, M.M. y Castillo-Gurrola, J., Estimation of the vulnerability to saline intrusion of the coast of Hermosillo aquifer, Sonora, Mexico, Geofísica Internacional, 43 (4), 611-621 (2004).

Roman-Calleros, J. y Ramírez-Hernández, J., Interdependent Border Water Supply Issues: The Imperial and Mexicali Valleys, in The US-Mexican border environment: Binational water management planning by Michel S., pp 95-144 San Diego State University Press, San Diego, California (2003).

Schroeder, R.A., Setmire, J.G. y Densmore, J.N., Use of stable isotopes, tritium, soluble salts and redox sensitive elements to distinguish groundwater from irrigation water in the Salton Sea area, in Irrigation and Drainage by Ritter F.W., pp 524-530 American Society of Civil Engineers, Honolulu, Hawaii (1991).

Tompson, A., Demir, Z., Moran, J., Mason, D., Wagoner, J., Kollet, S., Mansoor, K. y McKereghan, P., Groundwater Availability Within the Salton Sea Basin. 2008, Final Report. Lawrence Livermore National Laboratory. (en linea), https://e-reports-ext.IInl.gov/pdf/356709.pdf. Acceso: 6 de Noviembre (2012).

UNAM, Colecta, conservación y almacenamiento de muestras de agua. 2005, Manual de Geoquímica. CJuriquilla, Queretaro, México. centro de Geociencias, UNAM. (en linea), http://www.geociencias.unam.mx/geociencias/laboratorios/geoquimica/manual_geoq_ambiental.pdf. Acceso: 6 de Noviembre (2012).

Valenzuela-Vasquez, L., Ramírez-Hernández, J., Sol-Uribe, A. y Ruiz-Ortíz, S., Estudio hidrogeoquímico de las aguas subterráneas para uso agrícola en el área de riego del modulo uno en la margen izquierda del Río Colorado sobre terreno Mexicano, XII Congreso Internacional en Ciencias Agrícolas y Simposium en Agricultura Sustentable, 189-194, Mexicali, Baja California, México 29 al 30 Octubre (2009).

Vélez, O.M.V. y Rhenals, G.R.L., Determinacion de la recarga con isotopos ambientales en los acuíferos de Santa Fe de Antioquia, Boletin de Ciencias de la Tierra, 24, 37-54 (2008). 